\title{
Pregnancy Outcome in a Patient with Lipodystrophy and Type 2 Diabetes
}

Tolulope Shonibare, Muhammad Butt

Department of Diabetes and Endocrinology, Huddersfield Royal Infirmary UK, Peterborough City Hospital UK

\section{Introduction}

Familial partial lipodystrophy (FPLD) is characterised by a partial loss of subcutaneous fat in the limbs. It also results in excessive adipose tissue around the neck and face with variable involvement of the trunk ${ }^{1,2}$. It has an autosomal dominant inheritance pattern and is mostly associated with mutations in the LMNA gene (Lamin A/C) ${ }^{3}$. Two main variants have been described: the Kobberling (FPLD1) and Dunningham (FPLD2) variants both of which differ by the involvement of fat accumulation in trunk and face. Mixed phenotypes have also been described, resulting in some of the nomenclature referring them to as one entity ${ }^{4}$. FPLD share the clinical manifestations of metabolic syndrome such as insulin resistance, Acanthosis Nigricans, hypertriglyceridaemia and premature cardiovascular disease ${ }^{1-4}$.

In pregnancy this pathophysiological continnum of insulin resistance is compounded, making management a challenge. We present a case report of a patient which highlights these challenges.

\section{Background}

A 23 year old woman diagnosed with Dunnigan-type familial partial lipodystrophy (FPLD) attended the joint Antenatal / Endocrine clinic at 13 weeks of gestation. On examination she had a body habitus consistent with FPLD. She was diagnosed at age 7 and subsequently developed type 2 diabetes at age 11 years. She was managed initially with metformin followed by addition of insulin.

In addition, her past medical history included hypothyroidism, acanthosis nigricans, chronic pancreatitis, hypertension and mixed hyperlipidaemia. Her mother and sister were also diagnosed with the kobberling-dunningham variant lipodystrophy.

It was an unplanned pregnancy and her booking $\mathrm{HbA} 1 \mathrm{c}$ was $8.4 \%$ (IFCC $68 \mathrm{mmol} / \mathrm{mol})$. Prior to pregnancy, she was on a basal bolus regime of Levemir and Novorapid and her total daily insulin dose was approximately 180 units. At booking, we added Metformin 500mg twice daily, which was later increased to three times daily to improve insulin sensitivity.

Her insulin requirements escalated rapidly to a total daily dose of 250 units by 25 weeks gestation. At this stage, the use of Humulin R (U500) was considered and discussed with the patient. However, she presented with vaginal spotting and went into spontaneous labour at 26 weeks. She delivered a live female infant who was admitted to the special care baby unit. During labour she was managed with intravenous sliding scale insulin. On discharge, she continued on metformin and reverted to her pre-pregnancy regime of Levemir and Novorapid.

\section{Conclusion}

Familial Lipodystrophy is a group of rare disorders associated with numerous metabolic complications. Diabetes, familial lipodystrophy and pregnancy in combination confer a severe insulin resistant state which if poorly controlled, can have an adverse effect on pregnancy outcome.

In our patient her daily insulin requirements were rising exponentially, making it practically difficult to administer very large doses of insulin. Data on the use of U500 insulin during pregnancy is limited however there are a handful of case reports of its use in pregnancy with successful management of glycaemic and obstetric outcome ${ }^{5,6}$.

This case is a reminder of the challenges in the glycaemic management of patients with lipodystrophy particularly in pregnancy. It also adds to the limited literature available on pregnancy outcome of patients with lipodystrophy.

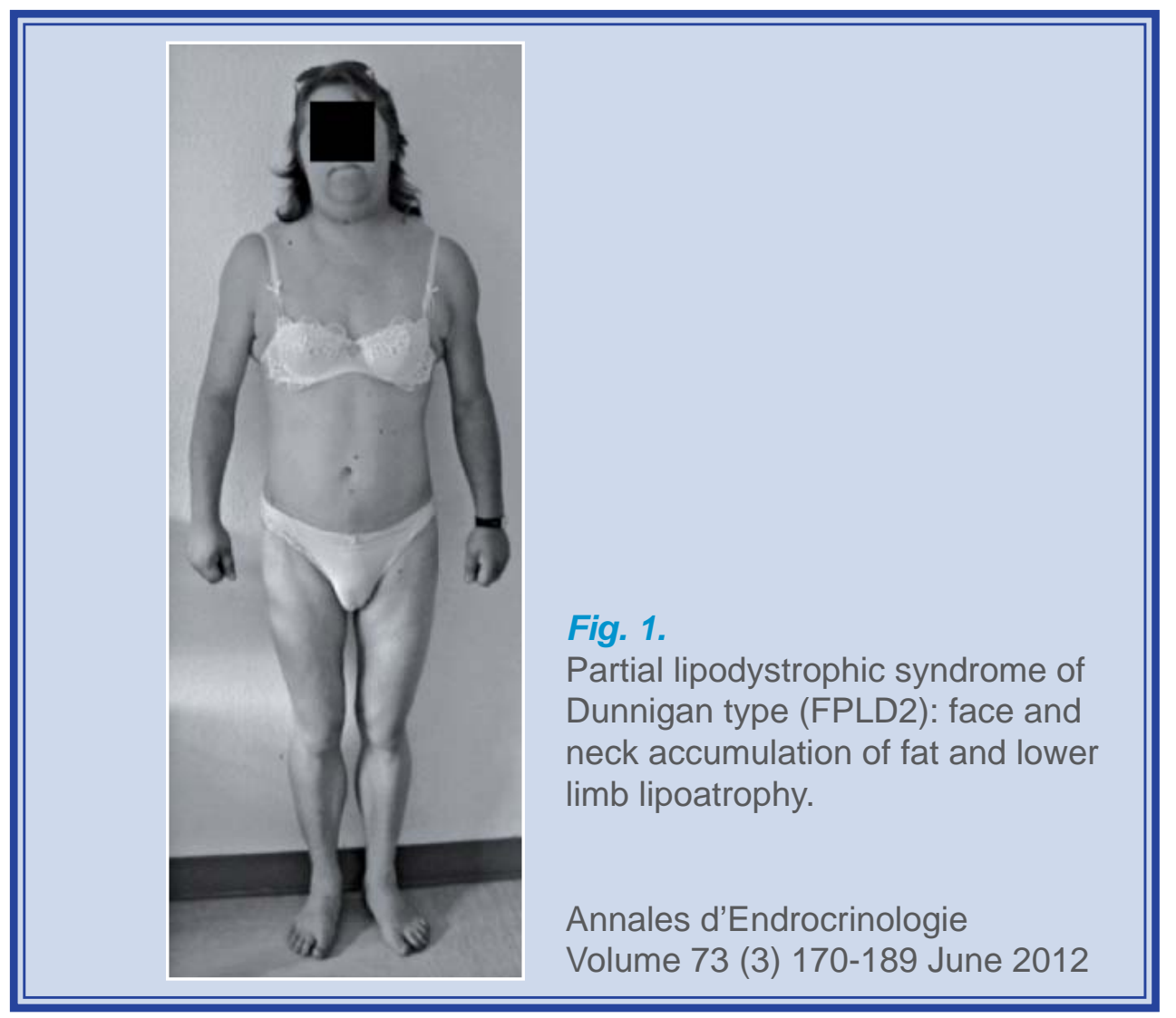

\footnotetext{
References

1. Köbberling J, Willms B, Katterman R, Creutzfeldt W: Lipodystrophy of the extremities: a dominantly inherited syndrome associated with lipoatrophic diabetes. Humangenetik 29:111-120, 1975

2. Köbberling J, Dunnigan MG: Familial partial lipodystrophy: two types of an X linked dominant syndrome, lethal in the hemizygous state. J Med Genetics $23: 120-127$, 1986

3. Herbst KL, Tannock LR, Deeb SS, Purnell JQ, Brunzell JD: Chait; A Köbberling type of familial partial lipodystrophy: an underrecognized syndrome. Diabetes Care. 2003 Jun;26(6):1819-24

4. Jackson SNJ, Howlett TA, McNally PG, O'Rahilly S, Trembath RC: Dunnigan-Kobberling syndrome: an autosomal dominant form of partial lipodystrophy. QJM 90:27-36,1997

5. Dolberg BK, Lenhard MJ: Successful outcome of pregnancy in a patient with generalized lipoatrophic diabetes mellitus. Endocr Pract. 2000 Jan-Feb;6(1):34-6.

6. Evans KM, Millward BA: Marked Insulin resistance in pregnancy. Pract Diab Int April 2006 23(3) 115-117 2000
} 